\title{
Azimuthal flows in hadron collisions from quark-gluon string re- pulsion
}

\author{
Igor Altsybeev ${ }^{1, \star}$ and Grigory Feofilov ${ }^{1, \star \star}$ \\ ${ }^{1}$ Saint-Petersburg State University
}

\begin{abstract}
Color flux tubes (quark-gluon strings), formed at early stages of hadron-hadron collisions, may overlap in case of sufficiently high densities and interact, producing longrange azimuthal correlations. In the hypothesis of repulsive interaction, each string may acquire, before the hadronization, the additional transverse boost, which is an efficient sum of all accounted string-string interactions. This modifies transverse momenta to the particles formed in string decay, leading to modification of event-wise observables, like azimuthal asymmetry of two-particle correlations, over a wide range of rapidity.

In this report we discuss results of Monte Carlo model with string repulsion, where efficient string-string interaction radius is introduced. We show that the effect of string repulsion can be the main dynamic origin of the elliptic flow and of the higher harmonics, which are reflected in the complicated structures observed in two-particle long-range correlation topology in nucleus-nucleus collisions at RHIC and at LHC.
\end{abstract}

\section{Introduction}

The very first proposals for studies of azimuthal and long-range correlations were formulated in [1]. It was emphasized that the data on the azimuthal asymmetry in soft multiparticle production may contain very non-trivial information. In [2] it was shown that anisotropies in transverse-momentum distributions provide an unambiguous signature of transverse collective flow in ultrarelativistic nucleusnucleus collisions.

The azimuthal anisotropy in two-particle correlation functions observed at RHIC and LHC is characterized by the Fourier flow coefficients [3, 4]:

$$
v_{n}=\left\langle\cos \left[n\left(\varphi-\Psi_{n}\right)\right]\right\rangle .
$$

Here $\varphi$ is the azimuthal angle of the particle, $\Psi_{n}$ is the angle of the initial state spatial plane of symmetry, and $n$ is the order of the harmonic. The planes of symmetry $\Psi_{n}$ are not known experimentally and the anisotropic flow coefficients are estimated from measured correlations between the observed particles. The second Fourier coefficient $v_{2}$ is called elliptic flow and has been studied in details in recent years in number of experiments. The first results on the elliptic flow of charged particles at midrapidity in $\mathrm{Au}+\mathrm{Au}$ collisions at $130 \mathrm{GeV}$ were reported by the STAR Collaboration at RHIC [5]. It was assumed that the observed azimuthal anisotropy appears at very early time and is caused by

\footnotetext{
${ }^{\star}$ e-mail: Igor.Altsybeev@cern.ch

${ }^{\star} \star$ e-mail: feofilov@hiex.phys.spbu.ru
} 
the pressure gradient and anisotropic expansion of the matter formed in the collisions $[2-4,6]$. The anisotropy effects were confirmed later in $\mathrm{Pb}+\mathrm{Pb}$ collisions at the LHC. For example, large values of elliptic flow at the LHC were observed by the ALICE Collaboration [7]. In addition to the elliptic flow, direct $\left(v_{1}\right)$, triangular $\left(v_{3}\right)$ and higher flow coefficients are also obtained [4]. It was established that the initial state spatial anisotropy is converted, due to some collective behaviour, to the momentum anisotropy.

In the experimental studies of flows, two main methods are usually applied. In the first method, flow coefficients are extracted from a two-particle angular correlations function, usually studied in terms of difference between two particles in azimuthal angle $\Delta \varphi$ and pseudorapidity $\Delta \eta$. Correlations can be studied either between 'trigger' and associated particles (which are taken from different $p_{\mathrm{T}}$ ranges or by some PID selection criteria) or just by taking all pairs of particles in an event (untriggered correlations). Another method is based on multi-particle cumulants using moments of the flow vectors [8], which allows to eliminate a contribution from correlations that are not related to the initial geometry (so-called non-flow).

A new set of striking phenomena was obtained in the two-particle correlation studies: the socalled "ridge" was first observed in $\mathrm{Au}+\mathrm{Au}$ collisions at $\sqrt{s_{N N}}=200 \mathrm{GeV}$ at RHIC [9-11]. Another puzzling experimental effect in the two-particle angular correlations was obtained later in the first measurement of the triangular $v_{3}$ and higher order harmonics of charged particle flow in $\mathrm{Pb}-\mathrm{Pb}$ collisions at $\sqrt{s}=2.76 \mathrm{TeV}[12,13]$ : a double-peak structure in the away-side azimuthal region was discovered in the most central events. It was shown in [12] that this structure can be naturally described by the anisotropic flow Fourier coefficients, in particular, by adding the higher harmonics. Detailed studies of $p_{\mathrm{T}}$, centrality and rapidity dependences of elliptic and higher order flow harmonics were performed by CMS [14], ATLAS [15] and ALICE [12, 16] at the LHC. Recent review on novel phenomena in particle correlations in relativistic heavy-ion collisions can be found in [17].

The azimuthal anisotropy in two-particle correlations in $\mathrm{Au}+\mathrm{Au}$ and $\mathrm{Pb}+\mathrm{Pb}$ collisions is generally related to manifestations of high-order flows and is interpreted as the result of a hydrodynamic expansion of the medium (of a nearly perfect liquid) formed in the collision. We have to mention, that contrary to this interpretation, the collective flow effects in relativistic ion collisions could be also explained without the hydrodynamic assumptions in so-called particle free streaming approach [18]. The last one was motivated by the fact that the effects, similar to the collective flow and associated with hydrodynamics, were not expected for elementary pp or $\mathrm{p}+\mathrm{Pb}$ collisions. Remarkable experimental observations were done by CMS in very high multiplicity proton-proton collisions at the LHC: a positive long-range correlation was reported between two particles produced at similar azimuthal angles, spanning a large range in rapidity [19]. Recent CMS results on multi-particle correlations in pp collisions show that this structure has collective nature [20]. The strong effects were also found in $\mathrm{p}+\mathrm{Pb}$ collisions [21]. Also, unexpectedly for light colliding systems, the existence of so-called double-ridge structures was found in $\mathrm{d}+\mathrm{Au}$ collisions at RHIC [22, 23] and in $\mathrm{p}+\mathrm{Pb}$ collisions at the LHC [24, 25]. These experimental findings produced a number of challenges to the existing theoretical approaches.

The characteristic feature of these azimuthal asymmetries of multi-particle angular correlations is that they are extending over a long range in rapidity. Causality then requires that these long-range correlations, if they exist, originate from the earliest times of the collision [26]. Therefore, we would like to mention here just briefly those models that are related intrinsically to the initial stages of the QGP formation and to the observed long-range rapidity correlation phenomena (one may see also the reviews [27] and [28]).

In the model of color glass condensate (CGC) the boost invariant Glasma flux tubes are considered [26] which produce particles isotropically with equal probability along the length of the flux tube. Interactions among those particles produces pressure leading to collective radial flow. The av- 
erage transverse velocity is obtained here from the blast wave fits to the RHIC data obtained by the PHENIX collaboration. Thus the common transverse expansion experienced by particles in a flux tube collimates the particles forming a ridge like structure extended in rapidity.

The string-like approach considers the quark-gluon strings, formed in the result of color exchange between partons of colliding hadrons, as particle emitting sources. These sources are stretched longitudinally between the beam remnants and at certain density in the transverse plane they may start to interact, forming clusters and producing some collective effects. One may mention here the string-fusion and percolation phenomena extensively studied in nucleus-nucleus collisions in [29, 30]. In [29] the string percolation phenomenology is compared to the CGC results on effective string or glasma flux tube intrinsic correlations, including the ridge phenomena and long-range forward-backward azimuthal correlations. Color string percolation model and its similarities with the CGC were discussed in [30].

In the theoretical analysis of long-range correlations there are two major factors that should be taken into account. In the string-like approach both are intrinsically present: (i) formation of the particle production sources extended in rapidity and (ii) certain initial anisotropy of configuration of these sources (color-flux tubes or strings) in the transverse plane. It is the last factor that might be responsible for the azimuthal anisotropy effects of transport of particles through the given nonuniform medium. Among these approaches are the recent studies [31-33] of the azimuthal anisotropy of two-particle correlation functions, observed in hadron collisions, that are based on the assumption of quark-gluon string formation at the very early stages of hadron collisions and further interaction of particles produced by these sources with the strong colour fields inside string clusters. It is shown by the direct Monte Carlo simulations [33] that the elliptic flow can be successfully described in the color string picture with fusion and percolation, provided the anisotropy of particle emission from the fused string is taken into account. Two possible sources of this anisotropy are considered, propagation of the string in the transverse plane and quenching of produced particles in the strong colour field of the string [33].

Another motivation to understand the dynamics of QGP formation in relativistic heavy-ion collisions can be found in the dual holographic approach (one may see review in [34]). These issues of holography and AdS/CFT duality are being intensively studied at present. The system of QCD strings is considered to be attractively interacting and collapsing, and it is viewed as a QCD analog to AdS/CFT black hole formation [35-37].

In string model approach, following general assumptions from the Regge phenomenology, it is considered that quark-gluon strings (color flux tubes) are formed between the partons of the colliding nuclei at the early stage of hadron-hadron collision. The number of these strings depends on the number of participating nucleons $\left(N_{\text {part }}\right)$ and on the collision energy. In case of sufficiently high density these strings may overlap and interact by attracting or repulsing,

Possible interaction of color strings (color flux tubes) in the form of repulsion or attraction and formation of flow harmonics in hadron collisions was first considered more then 3 decades ago in [1]. The exact type of string-string interaction is not known. The sign of string-string interactions depends on the direction of color fluxes [1]. This problem of the string-string interaction has not yet been systematically addressed till recently $[35,38,39]$. The magnitude of this interaction in string tension units was found to be small $\sim 10^{-1}-10^{-2}$ [39]. It is the collective effect of the large number of strings that is responsible for significant compression of the system in the transverse plane, leading in case of attractive interaction to implosion of such high-density configurations of strings [40].

Recently a new interest to interactions bewteen color flux tubes appeared, that was motivated both by the experimental findings of long-rage correlation phenomena in hadron collisions and by the progress in fitting the data in the framework of the various string models. They include Pomeron 
exchange approach, Lund model and MC event generators based on this model, the collectivity effects like color reconnection and string fusion and percolation phenomena, etc. One may conclude that the field of initial stages of hadron collisions, in particular, in string-like approach, is being extensively explored.

In the present study we continue our investigations [41-44] of the role of string density effects in the formation of azimuthal asymmetry of two-parrticle correlation functions in the case of string-string repulsion. The paper has the following structure. After the Introduction we describe in the Section 2 the approach and the main assumptions that are implemented in our MC model [42]. Two-particle correlation functions at different centralities and harmonic decomposition of the azimuthal correlation function, obtained in the MC model, are presented in Section 3. Results on the $p_{\mathrm{T}}$ spectra and masssplitting of the elliptic flow coefficients are presented in the Section 4. Discussion and conclusions are given in the last two sections.

\section{MC model with repulsive strings}

In our study we consider a simplified approach to string-string interaction mechanism for the case of repulsion. Basing on this hypothesis, a Monte Carlo (MC) model [42] was developed. In this MC model, final-state particles from A+A interactions are generated in each event in the following stages: Stage 1. Simulation of the nuclei.

Initial positions of the nucleons in nuclei are generated in accordance to Woods-Saxon distribution. Nuclei of $\mathrm{Pb}^{208}$ are considered, the Woods-Saxon radius is $6.62 \mathrm{fm}$, diffusion $=0.546 \mathrm{fm}$. Inside each nucleon partons are distributed in transverse $(x y)$ plane with 2D-Gauss law with $\sigma_{x y}=0.4 \mathrm{fm}$. Number of partons inside each nucleon is generated by Poisson law, where the mean number depends on a collision energy and is a model parameter. Two such a nuclei with some impact parameter are generated in each collision.

Stage 2. Simulation of strings configuration.

Interaction between colliding hadrons is implemented at the partonic level: partons from colliding nuclei can interact forming a qurk-gluon string, if the distance between partons in $x y$ plane is less then parton interaction distance $d=0.4 \mathrm{fm}$ (another model parameter). Additionally, there is a $3 \%$ probability for a string to be a result of "hard scattering" of partons to emulate of jet-like structures. Other $97 \%$ of the strings are considered to be "soft" and long in rapidity, occupying rapidity range $y \in(-4,4)$.

Stage 3. Repulsion of the strings.

At the next step of the system evolution, "soft" strings interact with each other. In the current MC model, interaction manifests itself as a repulsion. The repulsion mechanism is adopted from [1]. In our MC model, an efficient string-string interaction radius $R_{i n t}$ is introduced in the transverse plane. We consider this variable $R_{\text {int }}$ as a free parameter (it is different from the string radius $r_{0}$ which is often considered to be of about $\left.r_{0}=0.2 \mathrm{fm}\right) . R_{\text {int }}$ determines "interaction zone" around each string, these zones may overlap, and, due to string-string mutual repulsion, in case of high string density each individual string acquires significant transverse boost $\beta$ (see [1] and [42] for detailed mechanism). The mean value of the string boost is adjusted to be $\bar{\beta} \approx 0.65$ in central $\mathrm{Pb}-\mathrm{Pb}$ collisions.

Stage 4. Event final state: hadronization of the strings.

Each string breaks in several places into quark-antiquark pairs with the same exponentially distributed $p_{\mathrm{T}}$ and in opposite azimuthal directions. Quarks from neighbour string pieces then combine and form a meson (mostly pions and $\rho$ ). In the laboratory frame, all the particles originated from one string are boosted by the factor $\bar{\beta}_{\text {string. }}$. Due to the numerous string-string interactions taken into account, the initial configuration of quark-gluon strings could be transferred into the particles azimuthal flows. 
The modified version of the MC model will be used in Section 4 to consider spectra and mass ordering for the particles with different masses.

\section{Some features of MC model with string repulsion}

It was shown earlier [43] that the MC model with string repulsion is capable to reproduce the characteristic features of two-particle correlation functions obtained in peripheral, semi-central and central collisions of heavy ions for charged particles, if rather large string-string interaction radius $R_{\text {int }}=2 \mathrm{fm}$ is used (see Figure 1).

The pads in the Fig. 1 illustrate the onset of collectivity with the increase of string density when passing from peripheral to central A-A collisions. In peripheral events, a structure along $\Delta \varphi$ is visible, which is formed due to the $\rho^{0}$ decays into pions. One may see also, that the evolution of two-particle angular correlations with centrality and the relevant transition from low-density to the high density in $\mathrm{Au}-\mathrm{Au}$ collisions at $\sqrt{s_{\mathrm{NN}}}=200 \mathrm{GeV}$ is correctly reproduced. (Note that STAR variables $\Delta \rho / \sqrt{\rho_{\mathrm{ref}}}$ [22] are used here for two-particle correlations.)
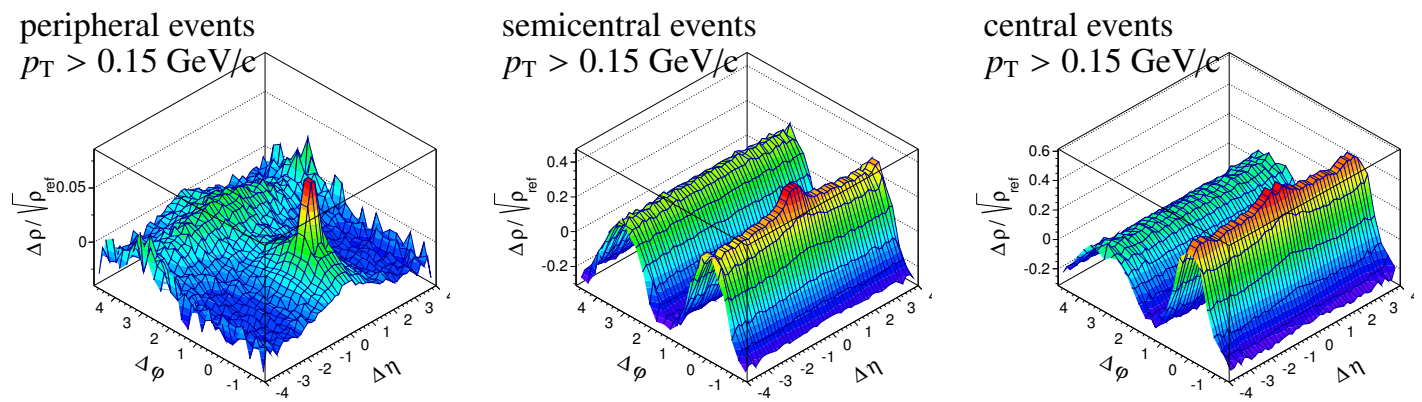

Figure 1. MC model [42] results for two-particle correlation functions obtained in the for peripheral (left), semicentral (middle) and central (right) events (from [43]). Particles with $p_{\mathrm{T}}>0.15 \mathrm{GeV} / c$ are analyzed. String-string interaction radius is $2 \mathrm{fm}$.

It was also obtained in [44] that two-particle azimuthal correlations, observed in very central $\mathrm{Pb}-\mathrm{Pb}$ collisions at LHC [12], can be obtained in the MC model with repulsive strings, along with the harmonic decomposition of the correlation function (see Figure 2). This decomposition reveals significant values of $v_{2}, v_{3}$ and $v_{4}$ coefficients.

\section{Transverse momentum spectra and mass ordering}

Another important experimental observation is a so-called mass ordering of flow harmonics, when $v_{n}\left(p_{\mathrm{T}}\right)$ points are "blue-shifted" to the right for hadrons with higher masses (see, for example, results for elliptic flow in $\mathrm{Pb}-\mathrm{Pb}$ collisions in [45]). It is natural that the $\mathrm{MC}$ model should be able to describe spectra and flow coefficients for different kind of particles. In the present work we check a dependence $v_{2}$ on $p_{\mathrm{T}}$ for pions, kaons and protons in order to see if the mass ordering is preserved.

For this study, string fragmentation in its rest frame was changed: pions, kaons and protons were generated as products of string decay (instead of just pions and $\rho$-mesons), and their transverse momentum spectra were parametrized by Tsallis formula tuned with data for pp collisions at $\sqrt{s}=7 \mathrm{TeV}$ [46]. Obtained spectra are shown in Fig.3 before application of string boosts (dotted lines), and after 


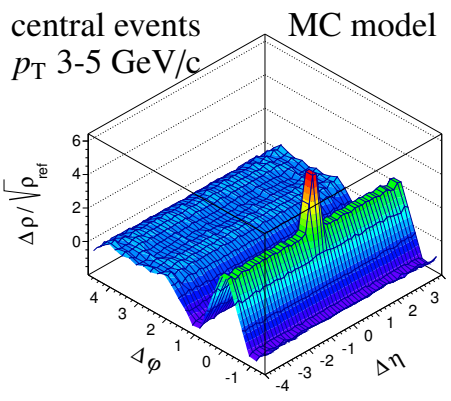

(a)

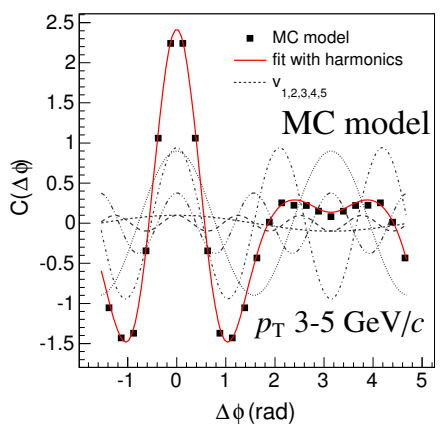

(b)

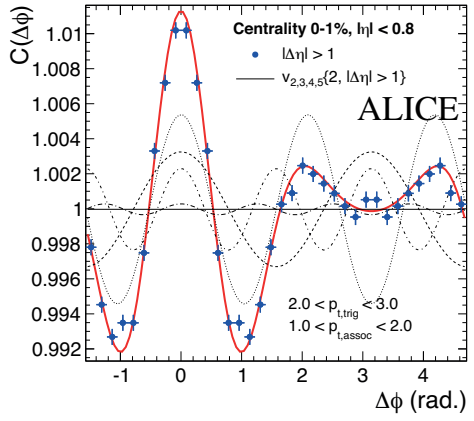

(c)

Figure 2. (a) MC model results for two-particle correlation function for very central nucleus-nucleus collision events for charged particles with $p_{\mathrm{T}}$ in $[3,5] \mathrm{GeV} / \mathrm{c}[42,44]$. String interaction radius is $2 \mathrm{fm}$. (b) Doublepeak around $\Delta \varphi=\pi$ in harmonics decomposition of two-particle angular correlations in MC model at the most central $\mathrm{Pb}-\mathrm{Pb}$ events $(b=0 \mathrm{fm})$ for charged particles with $p_{\mathrm{T}} \in[3,5] \mathrm{GeV} / c$. The solid red line shows the sum of the anisotropic flow Fourier coefficients $v_{1} \ldots v_{5}$ (dashed lines). (c) Experimental results [12] for harmonics decomposition of two-particle angular correlations in central $\mathrm{Pb}-\mathrm{Pb}$ collisions at the LHC. Note: the different definitions for two-particle correlation function are used at (b) and (c).

boosting (solid lines). Modifications of spectra for different types of particles are found to be in line with the observed hardening of $\left\langle p_{\mathrm{T}}\right\rangle$ observed for hadrons for more central $\mathrm{Pb}-\mathrm{Pb}$ events [47].

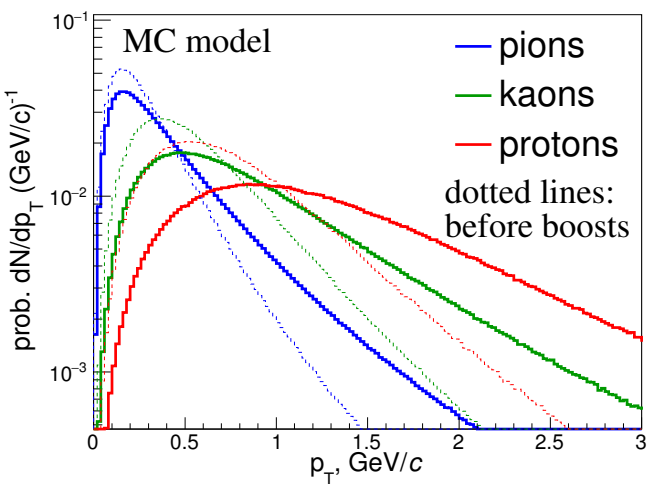

Figure 3. The $p_{\mathrm{T}}$-spectra for pions, kaons and protons in MC model before (dotted lines) and after string boosts (solid lines), in $\mathrm{Pb}-\mathrm{Pb}$ collisions with centrality $10-20 \%$.

To check the mass ordering, the $v_{2}\left(p_{\mathrm{T}}\right)$ was calculated with the Scalar Product (SP) method, similarly to what was done with $\mathrm{LHC} \mathrm{Pb}+\mathrm{Pb}$ data in [45]. Hadrons of one of the species ( $\pi, \mathrm{K}$ or $\mathrm{p}$ ) in $|\eta|<1$ were chosen as particles-of-interest, while reference charged particles were taken from $-3<\eta<-1$ and $1<\eta<3$, providing a pseudorapidity gap of $|\Delta \eta|>1.0$. This choice of rapidity ranges emulates experimental conditions of the $v_{2}\left(p_{\mathrm{T}}\right)$ analysis of identified hadrons in ALICE experiment [45]. The MC model results are shown in Fig.4 (b) and compared to data (a). It can be seen that the mass ordering observed in the experiment is qualitatively reproduced by the MC model in the region of interest (for $p_{\mathrm{T}}$ values below $3 \mathrm{GeV} / c$ ).

Is should be mentioned, that $v_{2}\left(p_{\mathrm{T}}\right)$ dependences for different particle species are quite sensitive to the shape of the $p_{\mathrm{T}}$ spectra, and parameterization of the spectra by Tsallis distribution tuned out to 
be good for reproduction of qualitative behaviour of the $v_{2}\left(p_{\mathrm{T}}\right)$. Another important point is that the effective string interaction radius $R_{\text {int }}$, which is the main parameter of the MC model, is still needed to be quite large - of about $2 \mathrm{fm}$.

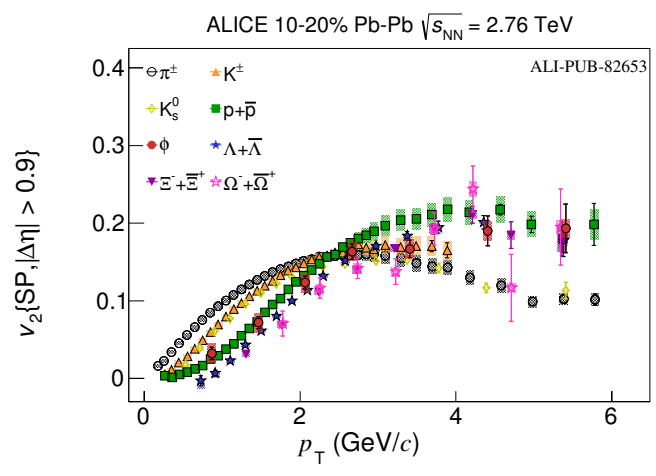

(a)

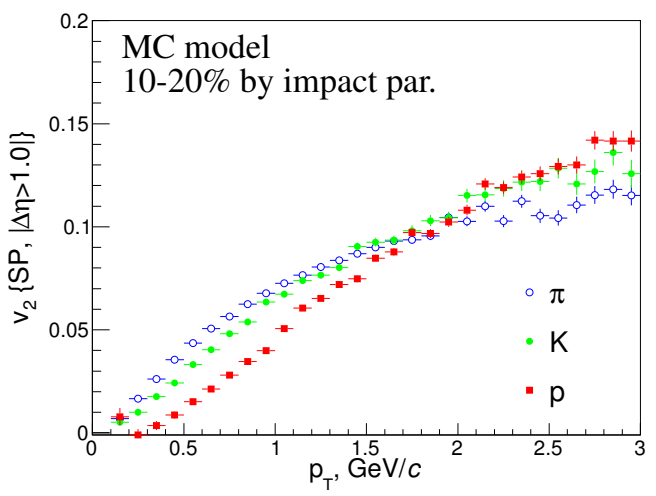

(b)

Figure 4. The $p_{\mathrm{T}}$-differential $v_{2}$ for pions, kaons and protons in centrality class $10-20 \%$ of $\mathrm{Pb}-\mathrm{Pb}$ collisions: (a) - ALICE data at $\sqrt{s_{\mathrm{NN}}}=2.76 \mathrm{TeV}$ [45], (b) $-\mathrm{MC}$ model calculations. The string interaction radius $R_{\text {int }}=$ $2 \mathrm{fm}$.

\section{Discussion}

Results of application of the MC model with the repulsive string-string interaction show that quite a large effective string-string interaction radius of about $2 \mathrm{fm}$ is needed in order to describe qualitatively (and in some cases almost quantitatively) azimuthal and rapidity topology of two-particle correlations picture, including the harmonic decomposition, centrality dependence of flow coefficients, mass ordering, etc. Possible physical mechanisms behind it could be the following:

(i) existence of rather weak (but long-range in transverse plane) interactions between color flux tubes (strings) mediated by exchange of some kind of meson, or

(ii) necessity to have a "dynamical" phase for a string when it moves within "string medium", before decaying into hadrons. It means that the string should experience some pressure from other strings (thus effectively increasing the interaction distances).

The current version of the MC model is based on simple kinematic assumptions and just a few parameters. There is still an open question: is it possible to describe pp and $\mathrm{p}$ - $\mathrm{Pb}$ effects with minor changes in model assumptions and parameter values? This check is be necessary to do if one wants to establish some uniform mechanism of flow generation.

\section{Conclusions}

High density string medium could be formed in hadronic collisions. It is shown in the Monte Carlo model that collective effects could appear due to some, rather weak, repulsive type of interaction between color flux tubes (strings). The particle-emitting sources (strings) are boosted by the combined repulsion by neighbours, thus the initial space anisotropy is converted into the anisotropic azimuthal distributions of charged particles. 
Similar results could occur at sufficiently high string density $\mathrm{p}-\mathrm{Pb}$ and $\mathrm{pp}$ collisions. More detailed quantitative analysis including mass ordering of flows in $\mathrm{p}-\mathrm{Pb}$ and $\mathrm{pp}$ collisions has to follow.

Acknowledgements. This work is supported by the Saint-Petersburg State University research grant 11.38.242.2015. The authors are grateful to V.Vechernin and V.Kovalenko for useful discussions.

\section{References}

[1] V.A. Abramovsky, O.V. Kanchely, JETP letters 31, (1980) 566.

[2] J.-Y. Ollitrault, PRD 46, 229 (1992), PRD 48, 1132 (1993).

[3] S. Voloshin and Y. Zhang, Z. Phys. C 70, (1996) 665.

[4] A. M. Poskanzer and S. A. Voloshin, Phys. Rev. C 58, (1998) 1671.

[5] K.H. Ackermann et al. (STAR Collaboration), Phys. Rev. Lett. 86 (2001) 402.

[6] Heinz Sorge, Phys. Rev. Lett. 78, (1997) 2309.

[7] ALICE Collaboration, Phys. Rev. Lett. 105, (2010) 252302.

[8] A. Bilandzic, R. Snellings and S. Voloshin, Phys.Rev. C83 (2011) 044913.

[9] F. Wang, STAR Collaboration, Talk at Quark Matter 2004, J. Phys. G 30 (2004) S1299-S1304.

[10] J. Adams, et al. (STAR Collaboration), Phys.Rev.Lett. 95 (2005) 152301.

[11] J. Adams, et al. (STAR Collaboration), Phys. Rev. C 73 (2006) 064907.

[12] ALICE Collaboration, Physics Letters B, Volume 708, Issues 3-5, (2012) 249-264.

[13] ATLAS Collaboration, Phys.Rev. C86 (2012) 014907.

[14] CMS Collaboration, PHYSICAL REVIEW C 87 (2013) 014902.

[15] ATLAS, Phys.Lett. B707 (2012) 330-348.

[16] ALICE Collaboration, arXiv:1605.02035, CERN-EP-2016-115 (2016).

[17] F. Wang, Progress in Particle and Nuclear Physics, 74 (2014) 35-54.

[18] P. Romatschkea, Eur. Phys. J. C (2015) 75:429.

[19] V. Khachatryan et al. [CMS Collaboration], JHEP 1009 (2010) 091.

[20] V. Khachatryan et al. [CMS Collaboration], arXiv:1606.06198 (2016).

[21] S. Chatrchyan et al. [CMS Collaboration], Phys. Lett. B 718, 795 (2013); Phys. Lett. B 724, 213 (2013).

[22] G.Agakishev et al. (STAR Collaboration), Phys.Rev.C 86, (2012) 064902.

[23] A. Adare et al. (PHENIX Collaboration), Phys. Rev. Lett. 111, (2013) 212301.

[24] B. Abelev et al. [ALICE Collaboration], Phys. Lett. B 719, 29 (2013); B. Abelev et al. [ALICE Collaboration, arXiv:1406.2474 [nucl-ex].

[25] G. Aad et al. [ATLAS Collaboration], Phys. Rev. Lett. 110, 182302 (2013); Phys. Lett. B 725, 60 (2013); The ATLAS collaboration, ATLAS-CONF-2014-021.

[26] A. Dumitru, F. Gelis, L. McLerran and R. Venugopalan, Nucl. Phys. A 810, (2008) 91.

[27] Wei Li, Mod. Phys. Lett. A27 (2012) 1230018.

[28] James D. Bjorken, Stanley J. Brodsky, and Alfred Scharff Goldhaber, arXiv:1308.1435 (2013).

[29] J. Dias de Deus and C. Pajares, Phys.Lett B695 (2011) 211-213.

[30] C. Pajares, Nucl. Phys, A 854 (2011) 125-130, arXiv:1007.3610.

[31] M.A.Braun and C.Pajares, Eur.Phys.J.C 71 (2011) 1558.

[32] M.A. Braun, C. Pajares, V.V. Vechernin, Nucl.Phys.A 906 (2013) 14.

[33] M.A. Braun, C. Pajares, V.V. Vechernin, Eur.Phys.J.A 51 (2015) 44.

[34] I. Arefeva, Phys. Usp. 57, (2014) 527. 
[35] E. Shuryak, arXiv:1412.8393 (2014).

[36] E. Shuryak and I. Zahed, arXiv:1311.0836 (2014).

[37] I. Iatrakis, A. Ramamurti and E. Shuryak, PHYSICAL REVIEW D 92, (2015) 014011.

[38] T. Kalaydzhyan, E. Shuryak, arXiv:1404.1888 (2014).

[39] T. Kalaydzhyan and E. Shuryak, arXiv:1402.7363 [hep-ph].

[40] T. Kalaydzhyan and E. Shuryak, arXiv:1503.05213 (2015).

[41] O.Kochebina, G.Feofilov, arXiv:1012.0173 (2010).

[42] I. Altsybeev, AIP Conf. Proc. 1701 (2016) 100002, arXiv:1502.03608.

[43] G.Feofilov, I.Altsybeev, O.Kochebina, AIP Conf. Proc. 1701 (2016) 060011.

[44] G.Feofilov, I.Altsybeev, O.Kochebina,PoS (Baldin ISHEPP XXII) 067 (2014).

[45] ALICE collaboration, JHEP 06 (2015) 190.

[46] ALICE collaboration, EPJC 75, (2015) 226.

[47] ALICE collaboration, Phys. Rev. C 88, (2013) 044910. 